CERN-PPE/95-85

24 May 1995

\title{
PROTOTYPE TESTS OF A 'JET CELL' DRIFT CHAMBER FOR LARGE-AREA MUON DETECTION
}

P. Bagnaia ${ }^{6)}$, E. Barberio ${ }^{3)}$, H. Beker ${ }^{6)}$, H. Bilokon ${ }^{4)}$, R. Bonini ${ }^{4)}$, A. Borisov ${ }^{5)}$, K. Bussmann ${ }^{2)}$, G. Capradossi $^{6)}$, J.L. Chevalley ${ }^{2)}$, V. Chiarella ${ }^{4)}$, G. Ciapetti6), M. Curatolo ${ }^{4)}$, G. Di Tore ${ }^{2)}$, M. Dris ${ }^{1)}$, B. Esposito ${ }^{4}$, C.W. Fabjan ${ }^{2)}$, T. Filippas ${ }^{1)}$, A. Franz ${ }^{2)}$, E. Gaumann ${ }^{2)}$, J.Ch. Gayde ${ }^{2)}$, E.N. Gazis ${ }^{1)}$, B. Goret ${ }^{2)}$, V. Goryatchev ${ }^{5)}$, W. Klempt ${ }^{2)}$, T. Kobayashi ${ }^{7)}$, S. Komamiya ${ }^{7)}$, A. Kozhin ${ }^{5)}$, C. Lasseur ${ }^{2)}$, D. Liguori ${ }^{3)}$, T. Mashimo ${ }^{7)}$, A. Nisati6), L. Passamonti ${ }^{4}$, M. Perciballi ${ }^{6)}$, C. Piscitelli6), L. Pontecorvo ${ }^{6)}$, J. Raynaud ${ }^{2)}$, E. Rosso ${ }^{2)}$, V. Russo ${ }^{4)}$, M. Schioppa ${ }^{3)}$, G. Susinno ${ }^{3)}$, G. Tentori ${ }^{4}$, A. Teodoli ${ }^{4)}$, M. Valdata Nappi ${ }^{3)}$, G. Viehhauser ${ }^{2)}$

\begin{abstract}
Large-area, high-precision drift chambers, based on the 'jet cell' concept, have been developed for the muon spectrometer of the ATLAS experiment at LHC. Emphasis is placed on the control and reduction of the systematic error in the drift measurement and in the mechanical construction. Results of measurements on prototypes are given. These indicate that a global precision of $\sigma \lesssim 70 \mu \mathrm{m}$ is achievable for large-area chambers.
\end{abstract}

1) Athens National Technical University, Athens, Greece.

2) CERN, Geneva, Switzerland.

3) Phys. Dep. Calabria University and INFN, Cosenza, Italy.

4) Laboratori Nazionali di Frascati dell' INFN, Frascati, Italy.

5) Institute for High Energy Physics, Protvino, Russia.

6) University 'La Sapienza' and INFN, Rome, Italy.

7) University of Tokyo, Tokyo, Japan. 
Experimentation at the planned Large Hadron Collider (LHC) at CERN will impose severe demands on all detector components. Most signatures of 'New Physics' will require identification and precision analysis of leptons, in particular of electrons and muons. The latter are privileged by the possibility of momentum analysis in the relatively low-rate environment behind the hadron calorimetry that will surround the central tracking system. Not surprisingly, the experiments planned for the LHC are influenced by the choice of the muon spectrometer $[1,2]$.

The muon precision tracking chambers will operate inside an air toroidal magnetic field, produced by eight very large ( $\sim 26 \mathrm{~m}$ long by $5 \mathrm{~m}$ wide) superconducting coils. The chambers, arranged in three layers to measure the sagitta of the muon tracks, have to operate in an inhomogeneous magnetic field, varying by more than $1 \mathrm{~T}$. The magnetic field in air was chosen to reduce multiple scattering to very low values $\left(\sigma_{p} / p \lesssim 0.02\right.$ at twenty to a few hundred $\mathrm{GeV} / c$ ). At very high momenta, the momentum resolution is determined by the measurement errors in the tracking system: in the ATLAS muon spectrometer the design goal of $\sigma$ (sagitta) $=70 \mu \mathrm{m}$ would result in $\sigma_{p} \sim 10 \%$ for a $1 \mathrm{TeV}$ muon. The approach of precision muon spectroscopy in air fields was pioneered for collider experiments by the L3 Collaboration at LEP [3]; it was also adopted by the GEM Collaboration for their experiment which was to be constructed at the Superconducting Super Collider (SSC) [4].

For the muon spectrometer of the ATLAS experiment [1] several technology options for the precision, i.e. momentum measurement, were pursued.

In this report we discuss one of these options, which uses a 'jet cell' as the basic detector cell. The 'Jet Cell drift chamber' is one of the most widely used technology for precision trackers [6]. We have adopted this concept to large-area planar drift chambers [7]. In Section 2 we explain the motivation for this choice; in Section 3 we comment on the engineering design developed for the ATLAS spectrometer and indicate construction procedures for the mass production of the precision chambers. The analysis of the errors which contribute to the systems resolution, are analysed in Section 4. Finally, we present test results obtained with a large, $4.3 \times 1.7 \mathrm{~m}^{2}$, prototype. Additional results can be found in Ref. [5].

\section{The Concept of the Jet Cell Chamber}

The ATLAS muon spectrometer will use $\sim 5000 \mathrm{~m}^{2}$ of precision chambers, arranged in three layers, with a performance goal of $\sigma_{\text {total }}<70 \mu \mathrm{m}$. The required acceptance in pseudorapidity is $\eta \lesssim 2.8$. Over most of this solid angle the count-rate is dominated by a collision-induced background of neutrons and photons and ranges from $\sim 20 \mathrm{~Hz} / \mathrm{cm}^{2}$ at $\eta \simeq 0$ to $>10^{3} \mathrm{~Hz} / \mathrm{cm}^{2}$ at $\eta \sim 2.8$. Furthermore, temperature variations in the experimental hall of a few ${ }^{\circ} \mathrm{K}$ and localized heat sources (electronics, power supplies) are to be expected.

Given these demanding performance and operation requirements, we aimed in the jet cell chamber (JCC) concept for reduction and control of possible systematic errors. This strategy applied to both of the two essential components: the detection cell and its support structure.

i) Detection cell: the jet cell is organized in 'bi-cells' and tilted with respect to infinitemomentum muons by $\theta_{T}=20.4^{\circ}$ (Fig. 1). In each cell, six to eight sense wires, alternating with potential wires, provide redundant measurements, which are statistically averaged. The tilt of the jet cell allows for 'auto-calibration' of the drift 
velocity $v_{D}$, the Lorentz angle $\theta_{L}$, and the offset time $t_{0}$. The 'local' measurements of these three critical parameters eliminate the major sources of systematic errors on wire accuracy in large systems. A further important measure in the control of single-wire accuracy is the use of saturated drift gases, which offer low sensitivity to gas composition, temperature, and pressure variations.

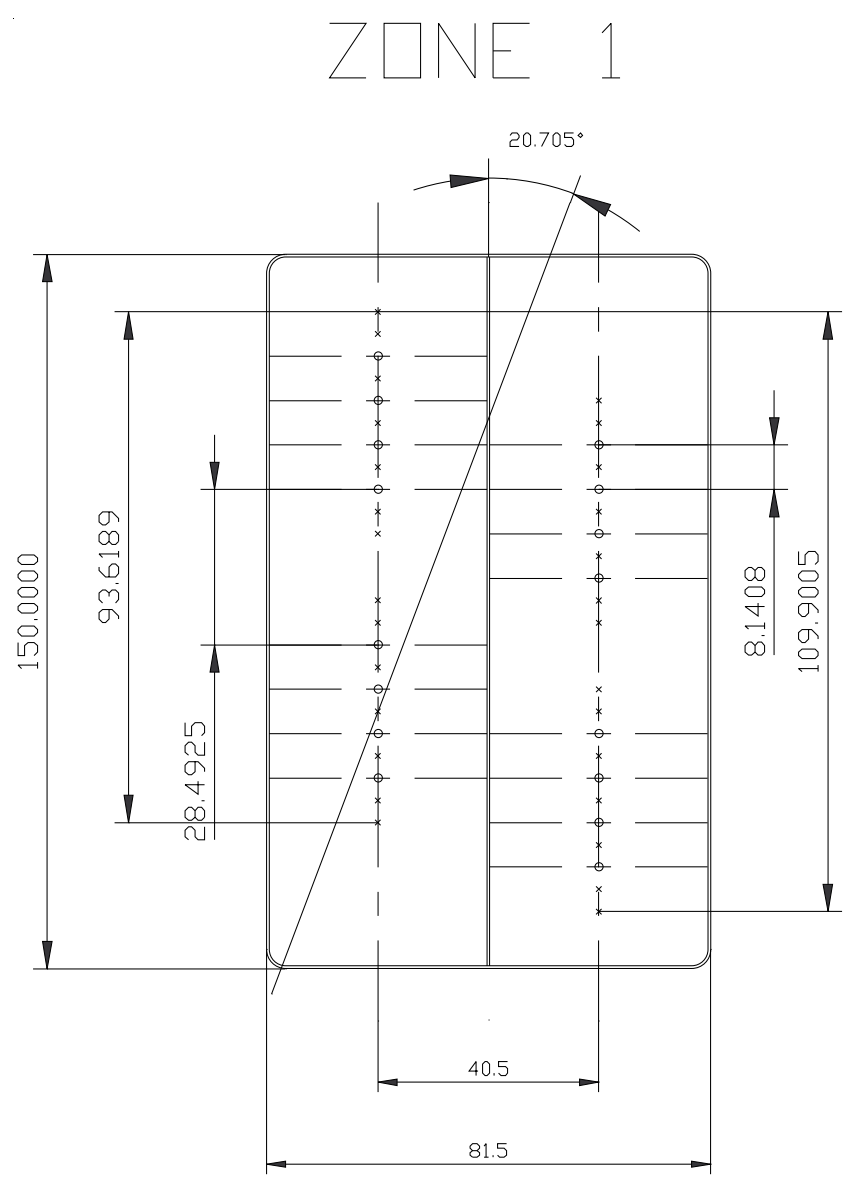

Figure 1: Layout of a bi-cell. The crosses indicate field wires and the open circles show the sense wires (dimensions are in millimetres).

ii) Support structure: support structures for the bi-cells were developed, which allow to position and maintain the jet cells with an accuracy of $\sigma \lesssim 30 \mu \mathrm{m}$ relative to global alignment references. The support is constructed from composite panels (Alhoneycomb with carbon-fibre facings on both sides). This choice provides for a light weight, yet rigid and temperature-stable support. Metallic brackets, glued to the surfaces, provide the precision location for the jet cells.

\section{The Jet Cell Chamber}

\subsection{The arrangement of the bi-cells}

The jet cells are arranged for construction purposes in bi-cells (Fig. 1). The six, seven or eight anode wires and potential wires in a cell are positioned with high precision at the ends by end flanges and are also precisely supported at approximately every $1 \mathrm{~m}$ by support combs. In this way the wires of a cell are localized to $\sigma<15 \mu \mathrm{m}$ relative to the locating holes of the end and support flanges. The arrangement of the wires inside 
a cell and the inclination of $\theta=20.7^{\circ}$ relative to infinite-momentum muons (see Fig. 2) is influenced by considerations of 'autocalibration'. Muons cross sense-wire planes or cell boundaries: the resulting constraint between the drift on the right and the left side of the wire plane is used to calibrate the drift velocity and Lorentz angle, as well as the zero-drift distance time $t_{0}$.

The overall layout of the chambers is shown in Fig. 2. The wire disposition inside a cell differs in four different polar-angle regions. This layout maximizes the number of sense wires activated by muons and guarantees that autocalibration is possible over the full acceptance. The sense wires are alternated with potential wires to reduce the cross talk and to provide control of the gas gain.

The conceptual design of the bi-cells was guided by the aim of using semi-automatic assembly procedures. The wire-supporting combs have an 'open' construction, thus allowing easy placement and fixation of the wires (Fig. 3). A relatively simple device strings all wires - the eight sense wires and the 14 potential wires - in one operation, as schematically indicated in Fig. 4. After stringing of the wires in both cells, the bi-cell is closed electrically and mechanically with two Al-shells (Fig. 5). It is a feature of this construction to use a set of a few, industrially-produced parts to build jet cells of the required length. With this method, bi-cells of up to $9 \mathrm{~m}$ length have been successfully fabricated.
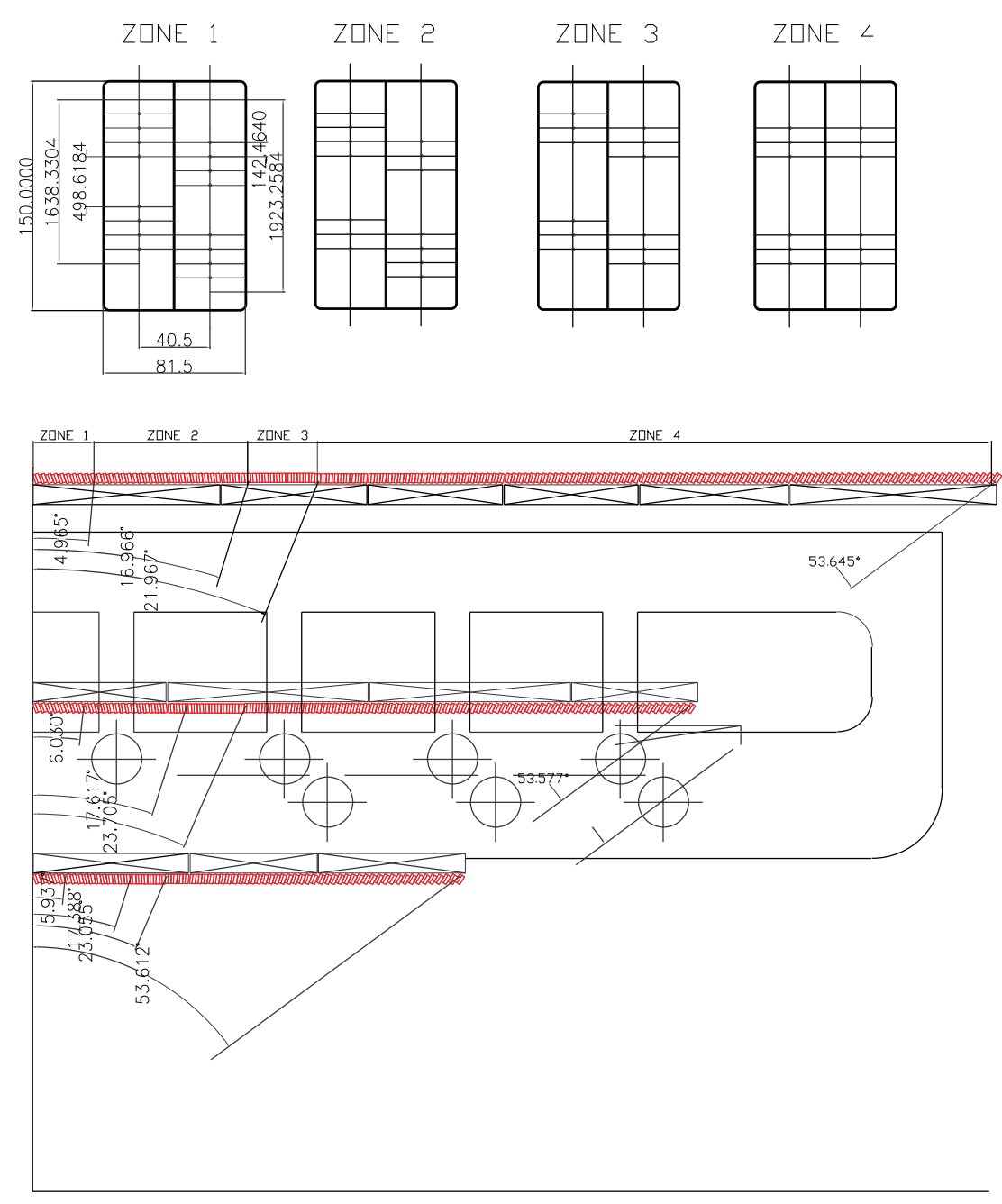

Figure 2: Layout of the jet cell wires in four zones of different polar angle; also shown is the arrangement of the JCCs in the toroidal muon magnet. 


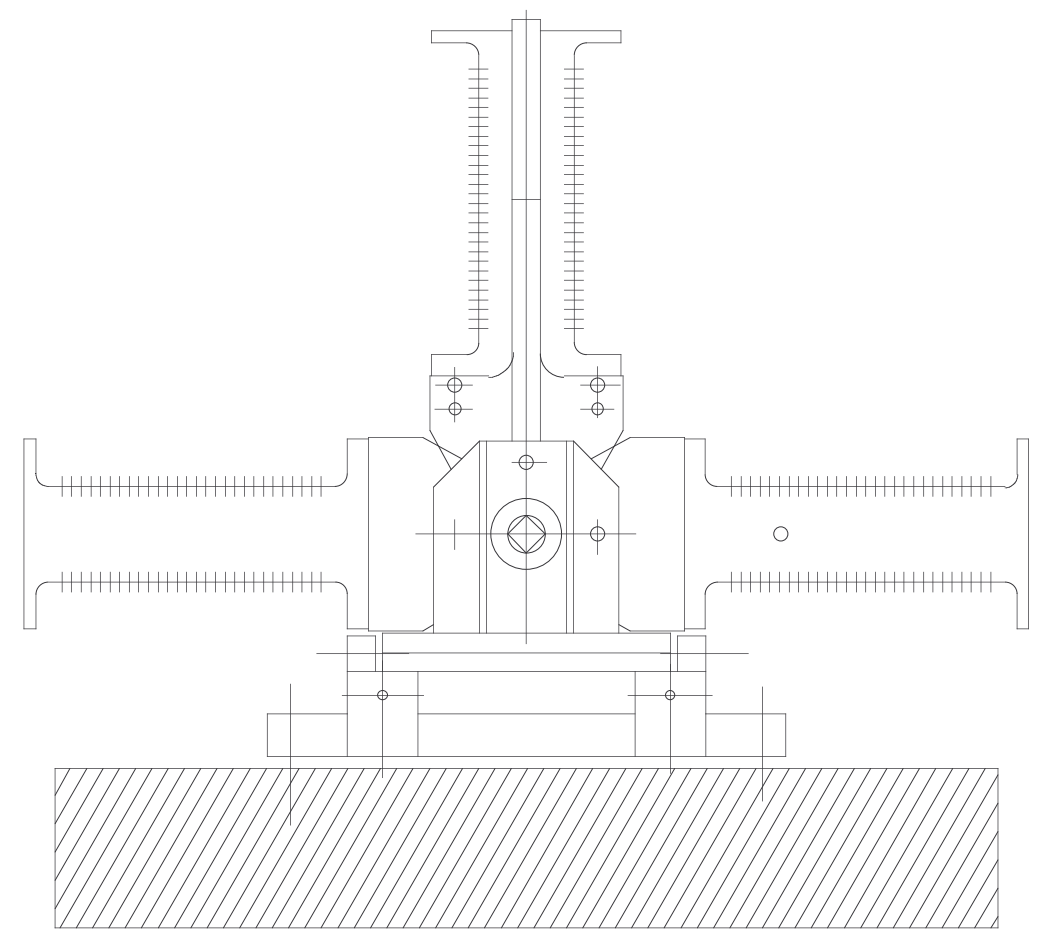

Figure 3: Wire-locating comb of a bi-cell mounted on a bi-cell construction bench; during the construction the combs are oriented in three different positions (all three positions are shown).
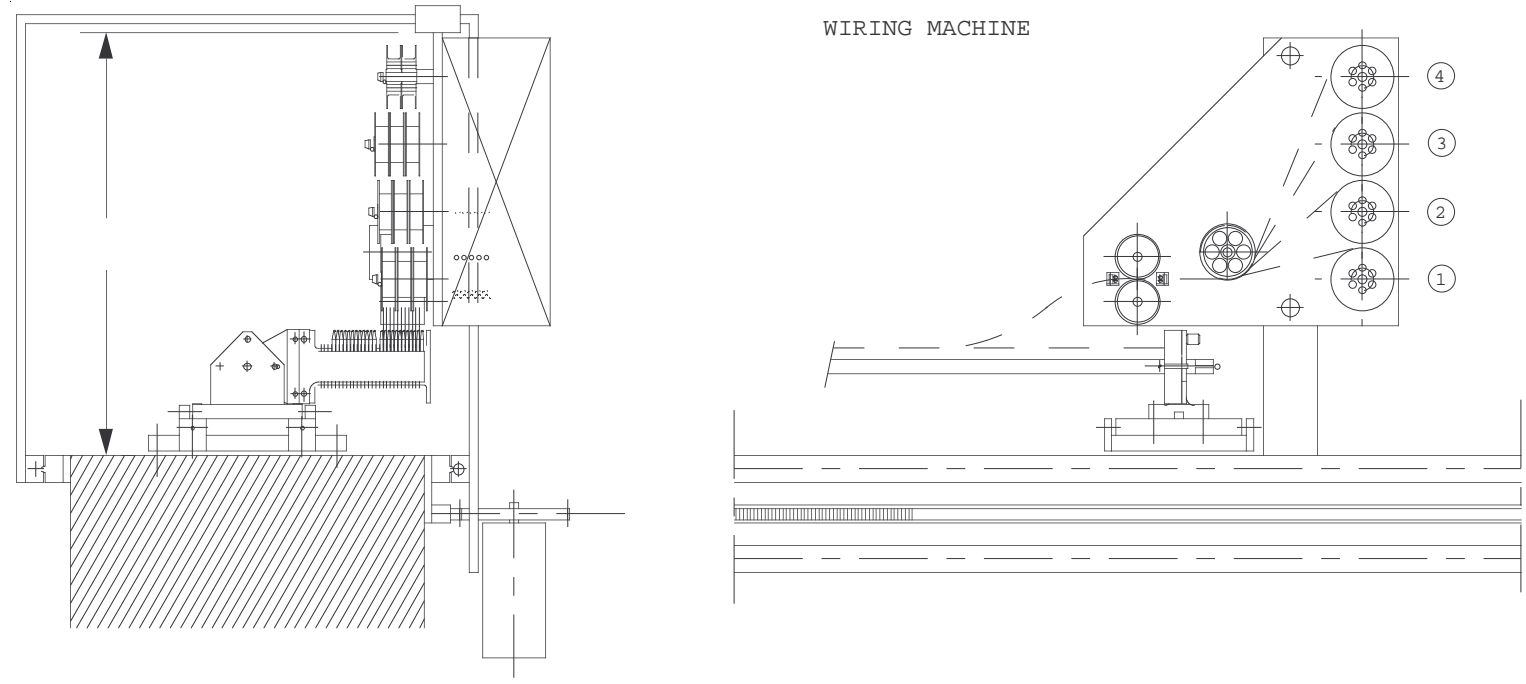

Figure 4: Three views of the wiring machine for the bi-cell wiring. All wires of a cell could be strung in one operation. 


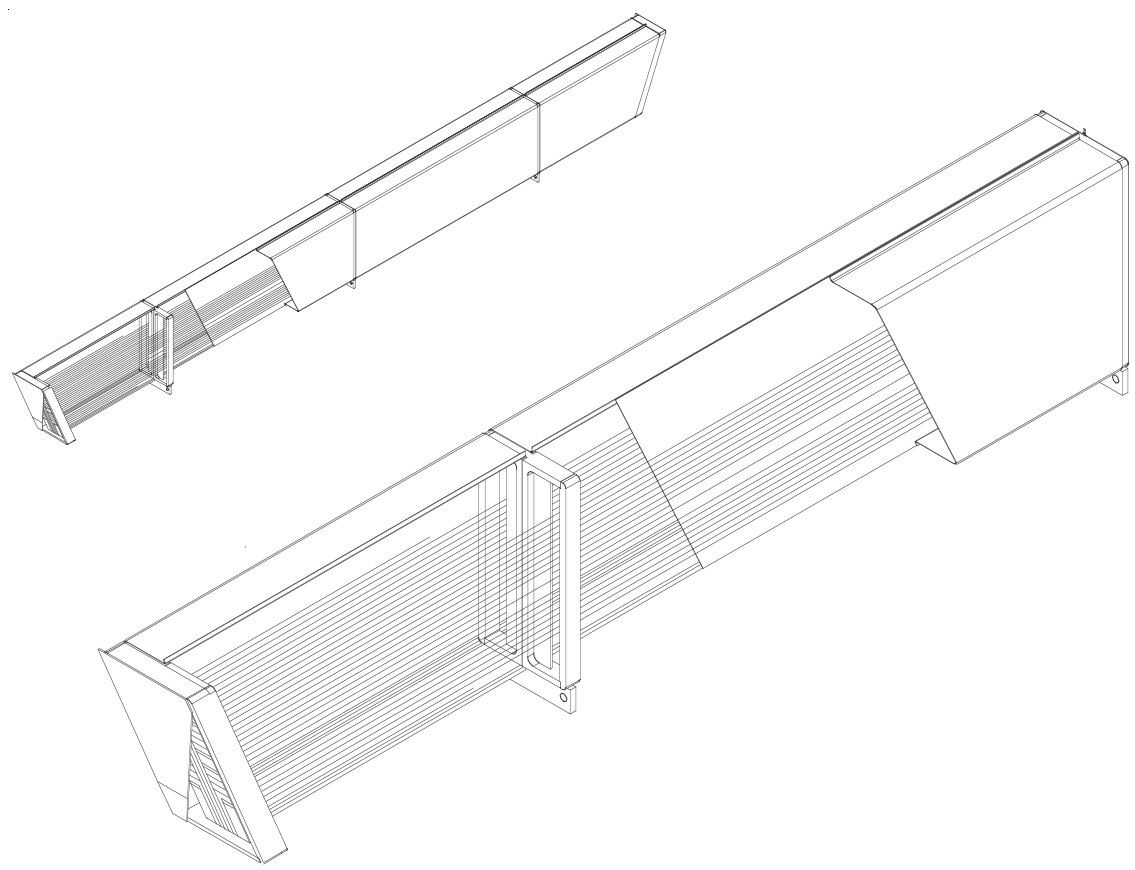

Figure 5: Schematic drawing of a bi-cell of $4 \mathrm{~m}$ length, corresponding to the design used for the prototype.

The single-cell parameters are summarized in Table 1.

Table 1: Single cell-parameter

\begin{tabular}{|ll|}
\hline Single-wire resolution $[\mu \mathrm{m}]$ & 120 (measured average) \\
Two-track resolution $[\mu \mathrm{m}]$ & 2500 (measured) \\
Gas mixture & $\mathrm{Ar} / \mathrm{CO}_{2} / \mathrm{C}_{2} \mathrm{H}_{6} 75 / 20 / 5$ \\
$v_{D}$ at operating voltage & $4 \mathrm{~cm} / \mu \mathrm{s}$ \\
Maximum drift time & $\sim 500 \mathrm{~ns}$ \\
$\theta$ (Lorentz) & $<12^{\circ}$ at $0.9 \mathrm{~T}$ \\
$d v_{D} / d$ pressure & $1.5 \times 10^{-4}$ per $1 \mathrm{~mm} \mathrm{Hg}$ \\
$d v_{D} / d$ temperature & $3.5 \times 10^{-4}$ per $1^{\circ} \mathrm{K}$ \\
Gas gain & $\leq 10^{5}$ (operating range) \\
Max. high voltage & $5 \mathrm{kV}$ \\
Wire material & $\mathrm{Cu}-\mathrm{Be}$; preferred also for sense wires \\
Wire diameter & $\sim 40 \mu \mathrm{m}$ for sense wires; \\
& $100 \mu \mathrm{m}$ for potential wires \\
Wire tension [\% of elastic limit] & $\leq 30 \%$ \\
Wire sag & $40 \mu \mathrm{m}$ max. \\
Method of wire location & injection moulded plates or combs \\
Neutron sensitivity & $2 \times 10^{-3}$ (no energy threshold) (calculated) \\
$\gamma$ sensitivity & $2 \times 10^{-2}$ (no energy threshold) (calculated) \\
Insensitive zone at chamber ends (plugs) & $1 \mathrm{~cm}$ (estimated) \\
\hline
\end{tabular}




\subsection{The JC support frame}

The support frames must locate the bi-cells with an accuracy of $\sigma<30 \mu \mathrm{m}$ relative to alignment references. We have evaluated a 'rigid' design, such that horizontally-placed chamber supports would not deflect by more than $\delta \lesssim 100 \mu \mathrm{m}$. This value was chosen to control the sagitta error due to this deflection in the intermediate polar angle $(\theta \lesssim$ $\left.70^{\circ},|\eta| \gtrsim 0.7\right)$ region. As an example we have analysed the largest structure required for the ATLAS spectrometer, Fig. 6.
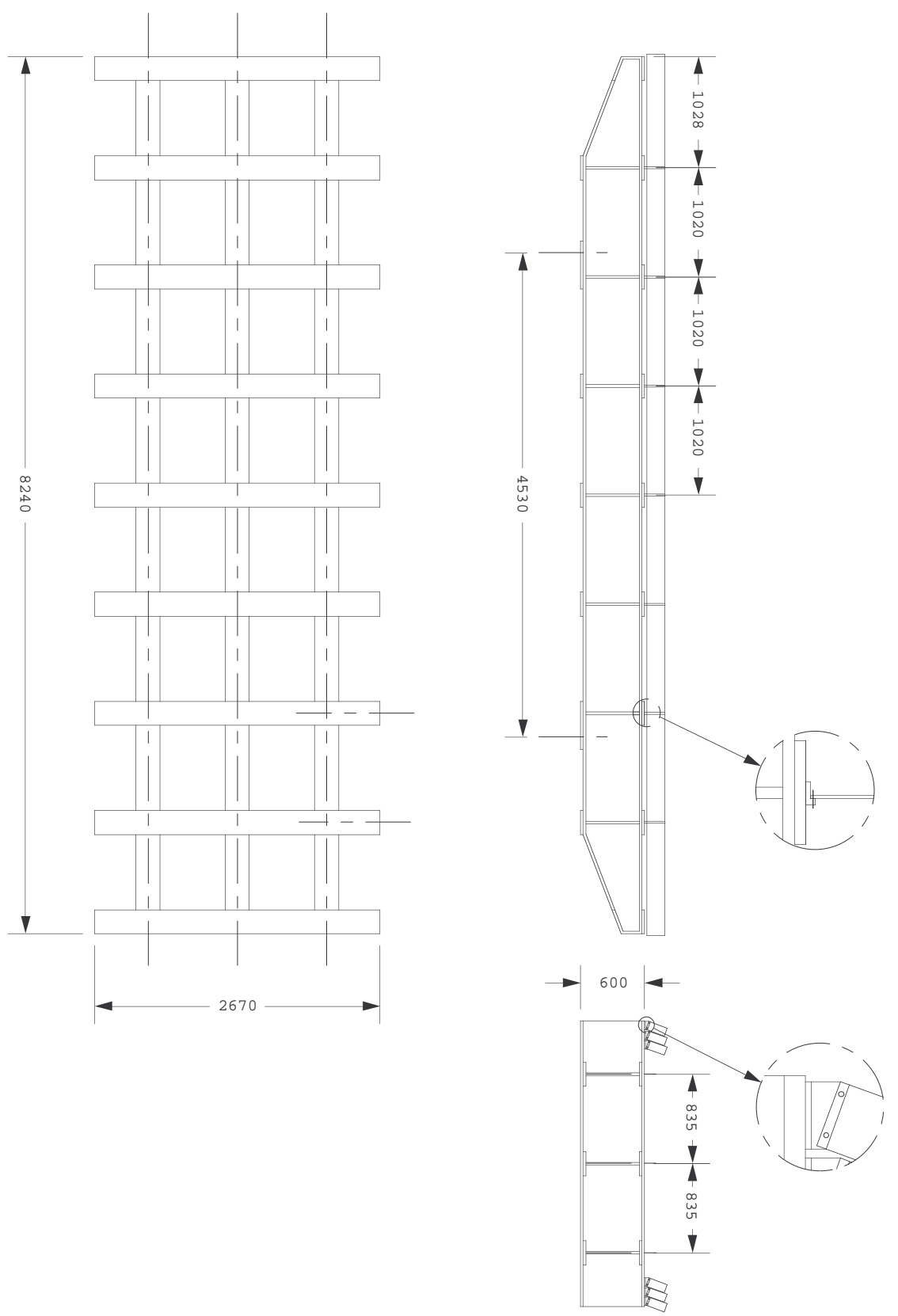

Figure 6: Drawing of the support structure. The dimensions correspond to chambers in the outermost layer of the toroid (dimensions in millimetres). When supported at the 'Bessel' points, the fully equipped structure will deflect by less than $130 \mu \mathrm{m}$.

The support is fabricated from rectangular composite-material sandwich plates. The core material is an $\mathrm{Al}$ honeycomb structure, $25.4 \mathrm{~mm}$ thick [density $\rho=60 \mathrm{~kg} / \mathrm{m}^{3}$ ]. The two skins are made from unidirectional carbon-fibre mats, each $1.2 \mathrm{~mm}$ thick. The module 
gets its stiffness from 12 vertical plates, 3 in lengthwise and 9 in widthwise direction. The module is closed by top and bottom plates to form rigid I-profiles together with the corresponding vertical plates.

Rows of brackets for mounting of the bi-cells are incorporated in the top plates running widthwise. After assembly of the structure to a moderate precision $( \pm 2 \mathrm{~mm}$ ), holes are drilled into the brackets using a drilling machine with a working surface of the dimensions of the module. These holes define the position of the bi-cells relative to the alignment references, which are also drilled at the same time. A precision of $\sigma=15 \mu \mathrm{m}$ has been achieved for this drilling operation.

A prototype with dimensions of $4.3 \mathrm{~m} \times 1.7 \mathrm{~m} \times 0.6 \mathrm{~m}$ has been manufactured by industry $^{1)}$ using this technique. Control of mechanical precision and the static deformation under load have been in complete agreement with the specifications and the finite element calculations (maximum deflection of $\sigma=130 \mu \mathrm{m}$ in the horizontal position).

The weight of this large support structure alone is approximately $190 \mathrm{~kg}$ and $440 \mathrm{~kg}$, when fully equipped with bi-cells. The thickness of equipped modules is approximately $0.05 X_{0}$ radiation lengths.

The chamber properties are summarised in Table 2

Table 2: Chamber and support properties

\begin{tabular}{|c|c|}
\hline Relative precision between wires & $<20 \mu \mathrm{m}$ (measured) \\
\hline Flatness of supports & $100 \mu \mathrm{m}$ (measured) \\
\hline $\begin{array}{l}\text { Precision of wires relative to } \\
\text { alignment references }\end{array}$ & $30 \mu \mathrm{m}$ (measured) \\
\hline $\begin{array}{l}\text { Procedures for aligning wires relative } \\
\text { to alignment references }\end{array}$ & by precision mechanical construction \\
\hline Uniformity of gas volume & open structure; therefore very uniform \\
\hline $\begin{array}{ll}\text { Thermal stability of support: } & \text { transverse } \\
\text { in plane }\end{array}$ & \} $10^{-6} /{ }^{\circ} \mathrm{K}$ \\
\hline Weight of chamber + support $/ \mathrm{m}^{2}$ & $\leq 18 \mathrm{~kg} / \mathrm{m}^{2}$ \\
\hline Radiation length of chamber + support & $5 \% X_{0}$ \\
\hline Average resolution per superlayer & $60 \mu \mathrm{m}$ \\
\hline
\end{tabular}

\section{$4 \quad$ Statistical and Systematic Errors}

One of the most important design considerations for the jet cell concept was the minimization and control of systematic errors. In this section we summarize the evaluation of systematic effects contributing to the error of the position measurement. The errors are expressed in r.m.s. deviations. All errors are based on measurements unless otherwise stated.

i) Wire positioning:

Wires are inserted in metallic holders with $\sigma=10 \mu \mathrm{m}$. The holders are positioned in the flanges relative to the flange locating holes with $\sigma=5 \mu \mathrm{m}$. The mechanical displacements between sense and potential wires will be further amplified by the electrostatic forces. For our operating conditions we calculate an amplification factor $F=1.5$. Consequently we assign a wire positioning error of

$$
\sigma_{\text {wire pos. }}=17 \mu \mathrm{m} \text {. }
$$

\footnotetext{
1) Manufactured by Fischer Advanced Composite Components Fischerstr. 9, Ried, Austria
} 


\section{ii) Gravitational sag:}

The wire tension is limited - for reasons of increased reliability - to $\lesssim 30 \%$ of the elastic limit. For $\mathrm{Cu} / \mathrm{Be}$ wires, supported every $1 \mathrm{~m}$, the maximum gravitational sag is $\delta=40 \mu \mathrm{m}$. The equivalent r.m.s. error is $\sigma_{\text {sag }}=13 \mu \mathrm{m}$. It will contribute to a sagitta measurement error (average of $\phi=2 \pi, 35^{\circ}<\theta<145^{\circ}$ ) of

$$
\left\langle\sigma_{\text {wire sag }}\right\rangle=3 \mu \mathrm{m} \text {. }
$$

iii) Bi-cell positioning:

The fixation holes have been positioned in the prototype relative to external alignment references with $\sigma=15 \mu \mathrm{m}$. The relative position error of the two holes determining the inclination of the bi-cell is measured to be $\sigma=5 \mu \mathrm{m}$, resulting in an equivalent average wire positioning error of $\sigma=8 \mu \mathrm{m}$. The combined effect is

$$
\sigma_{\mathrm{bi}-\mathrm{cell}}=17 \mu \mathrm{m} .
$$

iv) Mechanical deformations and $v_{D}$ variations:

The cathodes of the bi-cells are made from thin $(0.3 \mathrm{~mm}$ thick $)$ Al-sheets. From measurements made on the prototypes we infer a cathode flatness of $\sigma_{\text {cathode }}=0.3$ $\mathrm{mm}$. Assuming a saturated drift gas (Table 1 ) the resultant E-field and $v_{D}$ variations will contribute

$$
\sigma_{\text {mech.def. }}=3 \mu \mathrm{m} \text {. }
$$

v) Support deformations:

We have explained in Section 3 that the support structures were designed to be very stiff with maximum deformations $\delta \lesssim 130 \mu \mathrm{m}$. This sag will contribute to a sagitta error for muons with a polar angle $\theta \neq 90^{\circ}$. The average contribution $\left(35^{\circ}<\theta<\right.$ $\left.145^{0}, 0^{\circ}<\phi<2 \pi\right)$ to the sagitta error is found to be $\sigma_{\text {supp. sag }}=10 \mu \mathrm{m}$.. While this averaged error is quite small, it would be straightforward to apply corrections in parts of the detector, where the errors are relatively large.

vi) Temperature gradients:

Temperature-induced dimensional changes - approximately $120 \mu \mathrm{m} / \mathrm{m}$ for Al-structures and $\sim 5 \mu \mathrm{m} / \mathrm{m}$ for C-fibre structures for temperature variations $\Delta T=5^{\circ} \mathrm{K}-$ if caused by uniform temperature variations are planned to be tracked by the alignment system. Temperature gradients across the support are however of concern. For example, a gradient of $\Delta T=1^{\circ} \mathrm{K}$ across an $8 \mathrm{~m}$ long $\mathrm{Al}$ structure will induce deformations of $\sim 100 \mu \mathrm{m}$. We expect possible gradients of $\Delta T \sim 5^{\circ} \mathrm{K}$, resulting in deformations of a C-fibre support of $\sigma \sim 20 \mu \mathrm{m}$. The resulting sagitta error due to these temperature gradients is

$$
\sigma_{\Delta T}=5 \mu \mathrm{m} .
$$

vii) Summary of errors:

The stochastic measurement error per wire was found to be (Section 5) $\sigma=120 \mu \mathrm{m}$. Recognizing that, with the wire layout presented, an average of 9 wires per chamber are concerned in a measurement, we obtain after appropriate quadratic averaging of all errors mentioned

$$
\sigma(\text { chamber })=48 \mu \mathrm{m} .
$$

Assuming a relative alignment error of $\sigma=30 \mu \mathrm{m}$, the equivalent sagitta measurement error is found to be

$$
\sigma_{\text {sagitta }}=66 \mu \mathrm{m} .
$$


This error would permit a momentum measurement in ATLAS of $d p / p$ ( $1 \mathrm{TeV}$ muon $)$ $\approx 10 \%$.

\section{$5 \quad$ Test Results}

Measurements were carried out in the halo of the SMC muon beam at the CERN SPS and also with cosmic-ray muons.

The experimental set-up is schematically shown in Fig. 7. A $4.3 \mathrm{~m}$ long, $1.7 \mathrm{~m}$ wide C-fibre support prototype, instrumented with nine, $4.3 \mathrm{~m}$ long, bi-cells was exposed to muons. External reference precision drift tubes were also installed to provide a stable, external muon position measurement.

The principal aim of the test was a demonstration that the expected control of systematic errors can be achieved for chambers of a size typical for the ATLAS chamber dimensions. The beam test was not optimized to obtain the ultimate single-wire resolution.

The bi-cells were operated with an $\mathrm{Ar} / \mathrm{C}_{2} H_{6}$ (65/35) mixture.

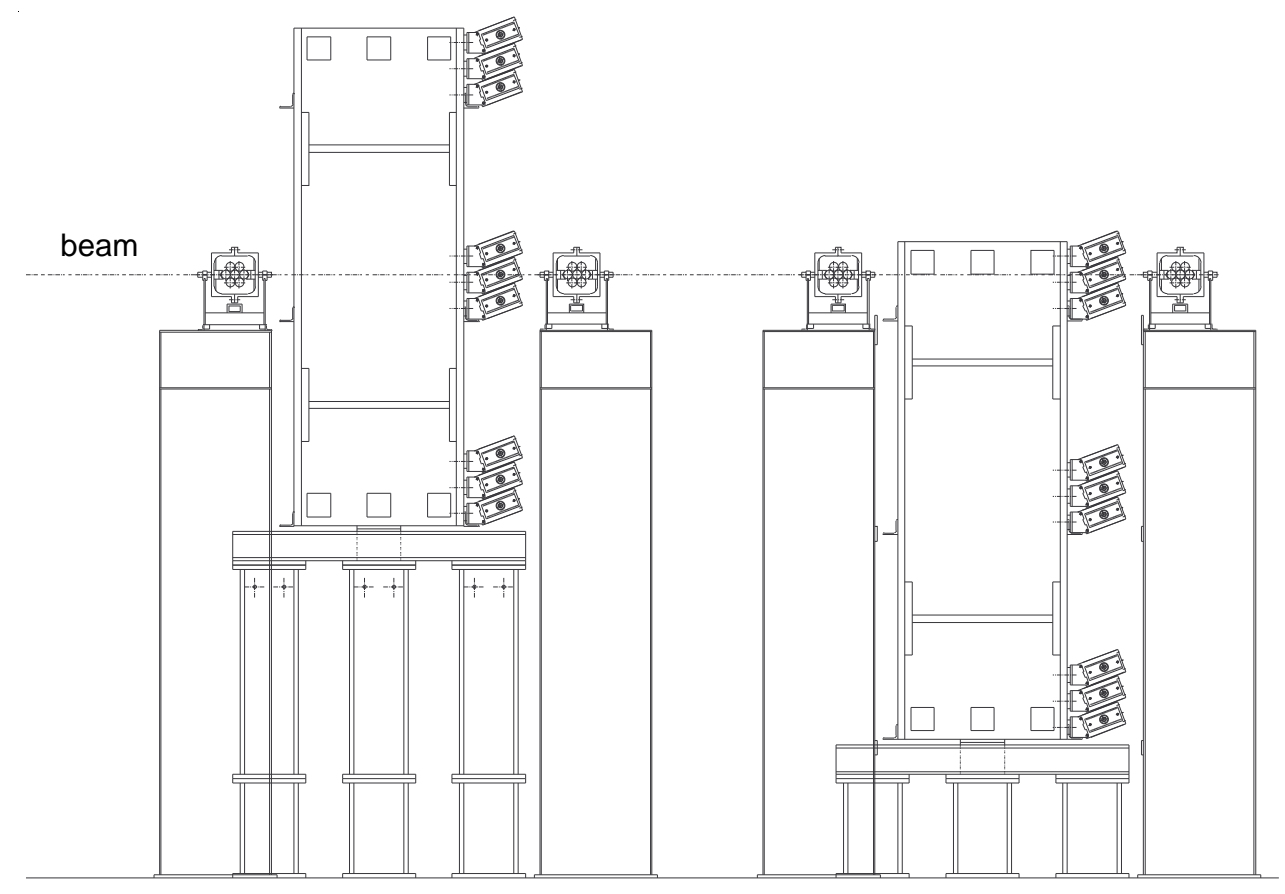

Figure 7: Layout of the JCC test area. The prototype was measured in two positions; drift tubes operated at $4 \mathrm{~atm}$ pressure were placed in front of and behind the JCC and provided an external reference measurement of the muons.

\subsection{Single-wire resolution}

The single-wire resolution was obtained from the distribution of the quantity $s=$ $y_{2}-\left(y_{1}+y_{3}\right) / 2$, where $y_{i}$ are the measured track distances to three neighbouring wires. The corresponding single-wire resolution $\sigma_{\mathrm{sw}}=\sqrt{2 / 3} \cdot \sigma_{s}$ is plotted in Fig. 8. A single-wire resolution, averaged over the drift distance of $d=2 \mathrm{~cm},\left(\sigma_{\mathrm{sw}}\right)=145 \mu \mathrm{m}$, was obtained. 




Figure 8: Single-wire resolution versus drift distance obtained in measurements in the test beam.

\subsection{Multi-track capability}

One of the attractive features of a uniform drift field geometry, as is typical for jet cell geometries, is the relatively good multi-track capability. This is of value for the detection of multi-hundred $\mathrm{GeV}$ muons, for which electromagnetic showering becomes important.

We have analysed a subset of data which contained more than one track in a jet chamber. The two-hit separation is shown in Fig. 9.

We extract good efficiency for multi-track reconstruction, provided the tracks are separated by more than $\Delta \approx 2.5 \mathrm{~mm}$.

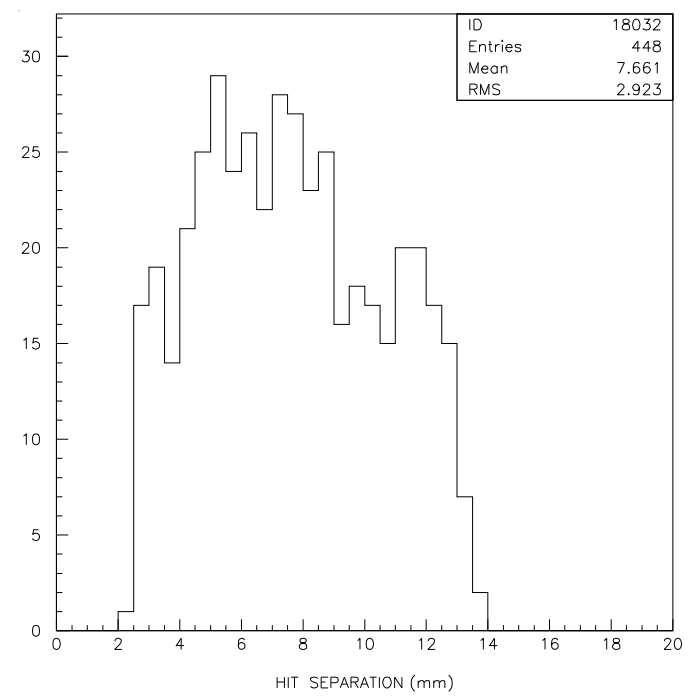

Figure 9: Two-hit separation recorded for a sample with two tracks in a jet chamber. Tracks are recognized if hits are separated by more than $\Delta \gtrsim 2.5 \mathrm{~mm}$. 


\subsection{Measurements with cosmic-ray muons}

Further studies were carried out with two prototypes using cosmic-ray muons. The two prototypes had a layout of wires very similar to the layout shown in Fig. 1, but differing in length: 0.50 and $9 \mathrm{~m}$ respectively. Furthermore, a gas mixture was used which could be a candidate for use in the actual experiment (Fig. 10). Its composition of $\operatorname{Ar}(75) / \mathrm{CO}_{2}(20) / \mathrm{C}_{2} \mathrm{H}_{6}(5)$ was determined with a view to obtaining a saturated drift velocity at relatively low electrical drift fields, and to combine a reasonable fast drift velocity with a moderately low Lorentz angle. The mean measured wire resolution was determined in two different ways. The residuals method used reconstructed tracks and a correction factor for the bias in the results due to the track fit; the sagitta method used the measured positions $y$ of three adjacent wires; this distribution characterized by an r.m.s. variation of $\sigma_{s}$ was then translated into a single-wire resolution $\sigma_{w}$ with the relation

$$
\sigma_{w}=\sqrt{\frac{2}{3}} \sigma_{s} .
$$

In Figure 11 the results of the two different prototypes are compared. The results of the $9 \mathrm{~m}$ chamber, for which no slewing correction was applied, are seen to be slightly worse. The improvement compared to the test-beam results is mostly due to better front-end electronics which became available for the cosmic-ray tests.

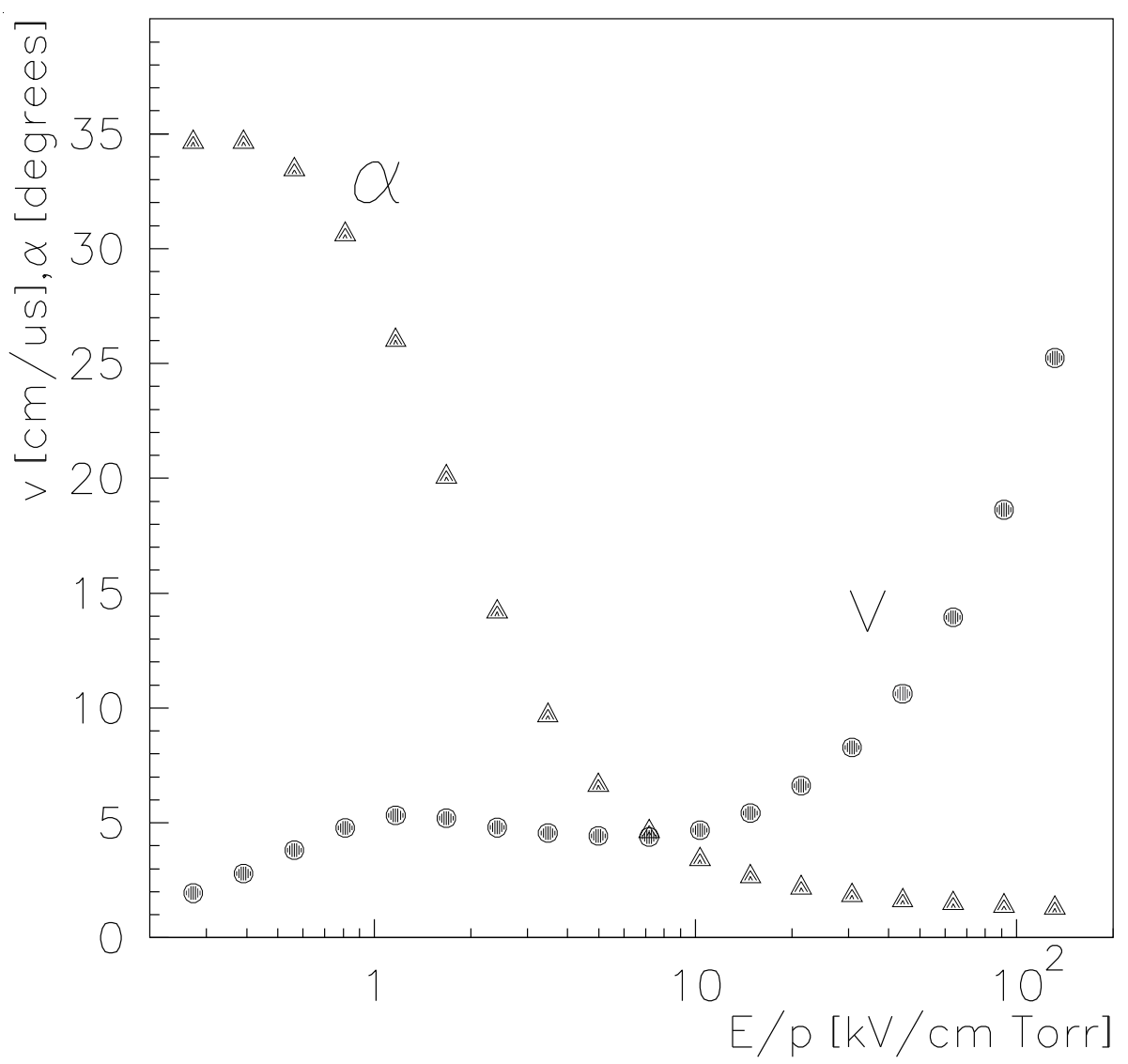

Figure 10: Calculated drift velocity (dots) and Lorentz angle $(\mathrm{B}=0.6 \mathrm{~T})$ (triangles) versus drift field for $\operatorname{Ar}(75) / \mathrm{CO}_{2}(20) / \mathrm{C}_{2} \mathrm{H}_{6}(5)$, as used in cosmic-ray tests. 


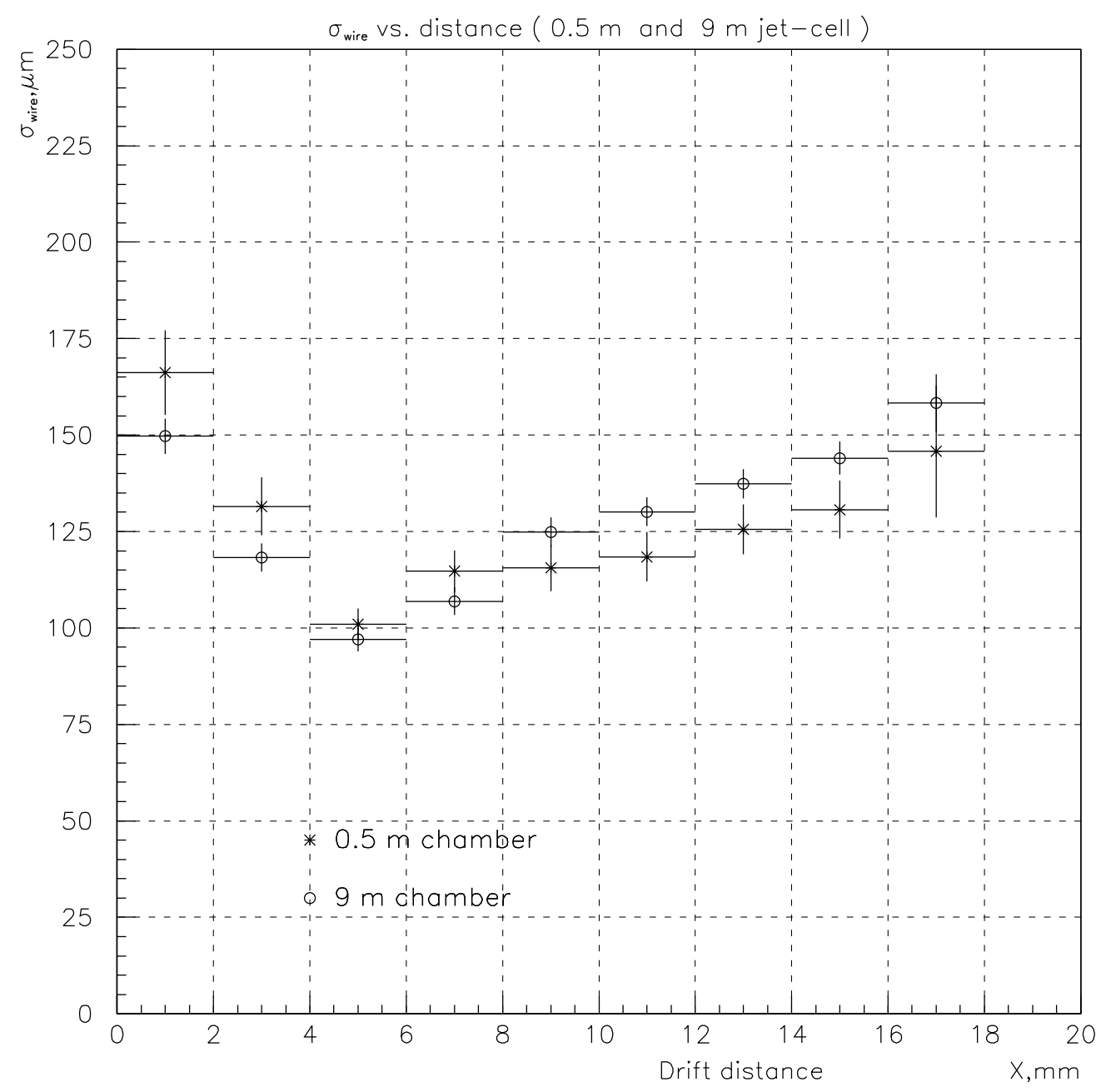

Figure 11: Single-wire resolution versus drift distance measured with the $0.5 \mathrm{~m}$ JCC (stars) and the $9 \mathrm{~m}$ JCC (circles).

\subsection{Absolute accuracy}

A major aim of the beam test was the measurement of the absolute position accuracy of the prototype and to demonstrate that the design goal of locating sense wires to better than $\sigma=50 \mu \mathrm{m}$ relative to reference marks can be achieved in large chambers. This measurement was carried out in the following way (see also Fig. 7). Measurements were taken at two distinct positions of the prototype by displacing the detector by a known distance $d=730.573(35) \mathrm{mm}$. This distance, the 'external' displacement, was measured with survey methods, external to the muon chamber. An 'internal' displacement was obtained by determining the coordinates of new sense wires relative to the precision reference (high-precision drift tubes), which were kept stable in time (e.g. by mounting the external references on quartz-plate supports). The 'internal' determination of the wire coordinates were inferred solely from the nominal mechanical positions of the bi-cells and the wires in a bi-cell. The difference between the external ('true') coordinate and the internal ('nominal') one was taken as a measure for the global accuracy of the prototype. This analysis gave:

$$
\text { position (wires, true) - position (wires, true) }=-59(63 \mu \mathrm{m}) \text {. }
$$


We consider this a good indication that within the errors $\sigma=63 \mu \mathrm{m}$ of this measurement the chamber satisfied our specifications.

\section{Conclusions}

Measurements on jet cell chamber prototypes have been reported. Using a nonflammable gas, we measure in the saturated drift mode single-wire resolutions of $\sigma \sim$ $120 \mu \mathrm{m}$, averaged over a $2 \mathrm{~cm}$ drift path. Measurements on a large prototype chamber indicate that global absolute accuracies of $\sigma \lesssim 50 \mu \mathrm{m}$ are achievable in our approach.

Central to the concept presented here is the control of systematic effects which are known to adversely affect the resolution of drift chamber systems. The approach discussed here is furthermore characterized by a minimum number of parameters which need to be monitored and by exceptional long-term stability. These features may be of critical advantage for very large precision systems, as are being planned for future collider detector facilities.

\section{Acknowledgments}

We are grateful to many colleagues in the ATLAS Collaboration who have shared their experience and knowledge with us. We gratefully acknowledge the financial and technical support made available to us by the Directors and Department Heads of the Collaborating Institutes.

\section{References}

[1] D. Gingrich et al., ATLAS Collaboration, Letter of Intent, CERN/LHCC 92-4 (1992).

[2] M. Markytan et al., CMS Collaboration, Letter of Intent, CERN/LHCC 92-3 (1992).

[3] B. Adeva et al., Nucl. Instrum. Methods A289 (1989) 35.

[4] W.C. Lefmann et al., Technical Design Report, GEM-TN-93-262 (1993).

[5] M. Dris et al., ATLAS Muon Detection Using Jet Cells, ATLAS Internal Note Muon - No 32 (February 1994).

[6] R.L. Wagner, Nucl. Instrum. Methods A265 (1988) 1;

R.D. Hener and A. Wagner, Nucl. Instrum. Methods 265 (1988) 11;

D. Saxon, Nucl. Instrum. Methods A 265 (1988) 20;

D.G. Cussans et al., Nucl. Instrum. Methods A315 (1992) 397.

[7] Y. Arai et al., Conceptual Design Report for the SDC Barrel and Intermediate Muon Detector Based on a Jet-type Drift Chamber, SDC-92-169 (1992). 